\title{
Editorial \\ The quest for a biomarker of circulating osteoclast precursors
}

\section{Christopher Ritchlin}

University of Rochester Medical Center, Rochester, NY 14642, USA

Corresponding author: Christopher Ritchlin, christopher_ritchlin@urmc.rochester.edu

Published: 17 June 2009

This article is online at http://arthritis-research.com/content/11/3/113

(C) 2009 BioMed Central Ltd

See related research by Lari et al., http://arthritis-research.com/content/11/1/R23
Arthritis Research \& Therapy 2009, 11:113 (doi:10.1186/ar2707)

\begin{abstract}
Osteoclast precursors arise from the CD14+ CD16- population in controls but details about cell surface marker expression and functional characteristics of these cells is unknown, particularly in patients with inflammatory arthritis. In a recent issue of Arthritis, Research and Therapy, Lari and colleagues found that osteoclasts developed from a proliferative CD14+ CD16- subset in healthy controls. These cells took on the morphology of osteoclasts, expressed mRNA for osteoclast-related genes and excavated pits on bone wafers. These findings provide new insights into monocyte diversity and provide evidence that osteoclast precursors arise from a small proliferating monocyte population in controls. Additional studies are needed in patients with inflammatory arthritis
\end{abstract}

"...Since (these cells) aside from their capacity to stretch out prolongations also are capable of consuming foreign bodies, we will subsume them under the joint name of fagocytes [sic]...."

Ellie Metchnikoff 1884

In a recent issue of Arthritis, Research and Therapy, Lari and colleagues provide evidence that osteoclast precursors arise from a novel subset of proliferating monocytes [1]. Research in this area originated with the seminal observations of Metchnikoff regarding the central importance of phagocytosis to human physiology, which culminated in a Noble Prize and laid the groundwork for the field of innate immunity at the opening of the last century [2]. Macrophages, pivotal effector cells in the innate immune response, maintain host defense, but also participate in wound healing and immune regulation [3]. In addition, precursor populations that differentiate into tissue macrophages exhibit heterogeneity in terms of surface marker expression and cytokine production [4]. Circulating monocytes have been divided into classical monocytes, which are CD14+ CD16-, and a small subset considered as non-classical monocytes, which are CD14+ CD16+ [5]. This latter population is increased in the circulation and synovial tissues of rheumatoid arthritis patients and these cells display an inflammatory phenotype characterized by increased release of interleukin-1 and tumor necrosis factor following exposure to lipopolysaccharide [6]. A unique subpopulation of CD14+ CD16- cells that exhibit a proliferative phenotype in vitro was identified by investigators in John Hamilton's laboratory and may represent an immature monocyte that has the ability to replicate in target tissues [7].

Circulating monocytes exhibit remarkable plasticity, being capable of differentiation into not only macrophages but also dendritic cells or osteoclasts in response to specific environmental signals [8]. Of particular interest is the finding that osteoclast precursors (OCPs) are elevated in the circulation of rheumatoid arthritis and psoriatic arthritis patients; in the case of psoriatic arthritis, elevated numbers of these cells correlate with joint damage and declined rapidly after patients were treated with anti-tumor necrosis factor agents [9]. In separate studies, OCPs were found to arise from the CD14+ CD16+ population [10].

Lari and colleagues [1] provide evidence that OCPs arise from a proliferative monocyte subpopulation in healthy controls. Previously, they reported that proliferative monocytic cells that express CD14, c-Fms, CD64 and CD33 but not CD16 give rise to osteoclasts in vitro based on an analysis of three healthy controls [10]. In the recent study [1], they analyzed monocytes from 13 healthy donors and demonstrated that osteoclasts were derived from the proliferative but not the non-proliferative fraction based on analysis of carboxyfluorescein succinimidyl ester (CFSE)-labeled cells. The authors state that functional analysis of proliferation may provide a better tool for identification of specific monocyte subsets since it is difficult to know if specific patterns of surface marker expression represent different states of activation or differentiation. 
The demonstration that OCPs derive from this proliferative monocyte population in controls is intriguing but must be interpreted with caution for several reasons. First, in vitro studies of monocytes can yield markedly different results depending on a variety of experimental variables, including cell density, serum concentrations and labeling conditions. To demonstrate the presence of this rare proliferative subset, cells were cultured for 9 days after labeling with CSFE. The expression data on osteoclast-related genes were obtained on day 23 of culture and osteoclasts were counted on day 30 . The fact that culture artifacts may obscure in vivo characteristics must be considered. Second, the cell surface phenotype was based on analysis of only three subjects, which weakens the premise that the parent population is CD16- given the high variability between subjects in expression of this marker. Lastly, it is highly likely that systemic (elevated production of tumor necrosis factor) and local (upregulation of RANKL (receptor activator for nuclear factor $\kappa B$ ligand)) events in patients with inflammatory arthritis dramatically alter the phenotype of circulating monocytes and these features are unlikely to be present in controls. Thus, alterations in monocyte populations obtained from healthy subjects may be considerably different from those observed in patients with inflammatory arthritis.

Despite these concerns, the importance of this proliferative subset in rheumatoid and psoriatic arthritis should be examined. If these proliferative monocytes prove to be expanded in arthritis and are progenitors of osteoclasts, several important questions need to be addressed. Are these precursor cells committed to the osteoclast lineage or can they differentiate into dendritic cells or macrophages? Are these cells CD16- as observed in controls or CD16+ as reported in psoriatic arthritis? Do they express higher levels of c-Fms on the cell surface as a mechanism to account for the increased proliferative capacity and lastly do they express unique cell surface markers? This last point is particularly important because the methods employed in these studies are not feasible for detection of this population by clinical laboratories due to the requirement for cell labeling and prolonged culture.

Increasing evidence points to a pivotal function for monocyte/ macrophages not only in synovial inflammation and joint destruction but also in obesity, eye and bowel inflammation and cardiovascular disease, which occur in many subjects with psoriasis and psoriatic arthritis [11]. Thus, it is crucial to understand if distinct monocyte precursor populations can differentiate into specific monocyte effectors because they may provide the much sought after susceptibility or response biomarkers for a number of different inflammatory disorders. The quest for these markers continues and this report highlights a novel population that may further clarify characteristic features of this elusive precursor population.

\section{Competing interests}

The author declares that they have no competing interests.

\section{References}

1. Lari R, Kitchener PD, Hamilton JA: The proliferative human monocyte subpopulation contains osteoclast precursors. Arthritis Res Ther 2009, 11:R23.

2. Kaufmann SHE: Immunology's foundation: the 100-year anniversary of the Nobel Prize to Paul Ehrlich and Elie Metchnikoff [see comment]. Nat Immunol 2008, 9:705-712.

3. Mosser DM, Edwards JP: Exploring the full spectrum of macrophage activation. Nat Rev Immunol 2008, 8:958-969.

4. Geissmann F, Jung S, Littman DR: Blood monocytes consist of two principal subsets with distinct migratory properties [see comment]. Immunity 2003, 19:71-82.

5. Passlick B, Flieger D, Ziegler-Heitbrock HW: Identification and characterization of a novel monocyte subpopulation in human peripheral blood. Blood 1989, 74:2527-2534.

6. Baeten D, Boots AM, Steenbakkers PG, Elewaut D, Bos E, Verheijden GF, Berheijden G, Miltenburg AM, Rijnders AW, Veys EM, De Keyser F: Human cartilage gp-39+,CD16+ monocytes in peripheral blood and synovium: correlation with joint destruction in rheumatoid arthritis. Arthritis Rheum 2000, 43:12331243.

7. Finnin M, Hamilton JA, Moss ST: Characterization of a CSFinduced proliferating subpopulation of human peripheral blood monocytes by surface marker expression and cytokine production. J Leukocyte Biol 1999, 66:953-960.

8. Miyamoto T, Ohneda O, Arai F, Iwamoto K, Okada S, Takagi K, Anderson DM, Suda T: Bifurcation of osteoclasts and dendritic cells from common progenitors. Blood 2001, 98:2544-2554.

9. Ritchlin CT, Haas-Smith SA, Li P, Hicks DG, Schwarz EM: Mechanisms of TNF-alpha- and RANKL-mediated osteoclastogenesis and bone resorption in psoriatic arthritis. J Clin Invest 2003, 111:821-831.

10. Komano Y, Nanki T, Hayashida K, Taniguchi K, Miyasaka N: Identification of a human peripheral blood monocyte subset that differentiates into osteoclasts. Arthritis Res Ther 2006, 8:R152.

11. Ritchlin CT: From skin to bone: translational perspectives on psoriatic disease. J Rheumatol 2008, 35:1434-1437. 